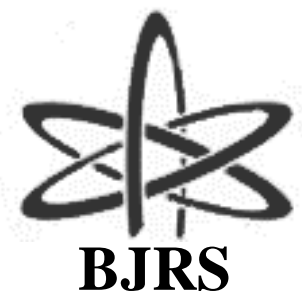

BRAZILIAN JOURNAL

$\mathrm{OF}$

RADIATION SCIENCES

06-03 (2018) 01-21

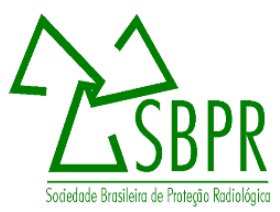

\title{
Análise das tecnologias e doses glandulares médias em mamografia no Brasil no período de 2011 a 2016
}

\author{
M. A. Pinheiro ${ }^{\text {a }}$ C. D. Almeida ${ }^{\text {a }}$ J. E. Peixoto ${ }^{\text {b }}$ M. A. S. Valverde ${ }^{\text {c }}$ A. V. Marin ${ }^{b}$ \\ ${ }^{a}$ Divisão de Física Médica, Instituto de Radioproteção e Dosimetria, CEP 22783-127, Rio de Janeiro-RJ, Brasil \\ ${ }^{\mathrm{b}}$ Instituto Nacional de Câncer (INCA), CEP 20231-092, Rio de Janeiro, RJ, Brasil. \\ ${ }^{\mathrm{c}}$ Centro de Saúde Coletiva Professor Manoel José Ferreira, CEP 25625-090, Petrópolis, RJ, Brasil.
}

claudio@ird.gov.br

\begin{abstract}
RESUMO
A mamografia apresenta risco de indução de câncer associado à dose glandular média (DGM), definida como a dose absorvida no tecido glandular da mama. No Brasil, o monitoramento dessas doses é realizado pelos programas de qualidade em mamografia do Colégio Brasileiro de Radiologia e do Instituto Nacional de Câncer. Este trabalho teve como objetivo analisar o impacto das tecnologias nas DGMs em serviços de todo o país entre 2011 e 2016. Foram analisados dados de 1.115 avaliações de DGM obtidas por meio da exposição de simuladores de PMMA para uma mama de $53 \mathrm{~mm}$ de espessura, em 845 mamógrafos de 716 serviços em 279 cidades. Os mamógrafos são de 13 fabricantes, sendo 192 de tecnologia com sistema filme-tela intensificadora (SFT), 544 de tecnologia digital computadorizada (CR) e 109 de tecnologia digital direta (DR). A média da dose glandular

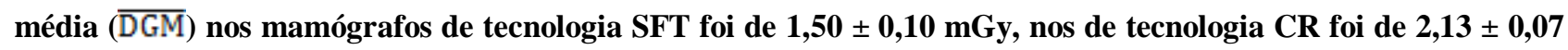
mGy e nos de tecnologia DR foi de 2,07 $\pm 0,20 \mathrm{mGy}$. Houve diferença estatisticamente significativa $(p<0,05)$ entre as $\overline{\mathrm{DGM}}$ s das tecnologias SFT e as digitais CR e DR. Entretanto, não houve diferença estatisticamente significativa $(p=0,70)$ entre as $\overline{D G M}$ s das tecnologias digitais CR e DR. Em 22,1\% das avaliações, as DGMs estavam acima do valor aceitável de 2,50 mGy, variando entre $11,6 \%$ na região Sul e 31,4\% na região Nordeste. Foi verificado que o aumento destas não conformidades ao longo do tempo apresenta relação direta com o aumento do número de mamógrafos de tecnologia digital.
\end{abstract}

Palavras-chave: Dose glandular média 1, Mamografia digital 2. 
ABSTRACT

Mammography has a risk of radiation-induced cancer associated with the mean glandular dose (MGD) defined as the absorbed dose by the glandular tissue of the breast. In Brazil, the Brazilian College of Radiology (CBR) and the National Cancer Institute (INCA) control these doses through their quality programs in mammography. The aim of this study is to analyze the impact of technologies on the MGD in mammography services throughout the country between 2011 and 2016. It was analyzed data from 1,115 DGM evaluations obtained through the exposure of PMMA phantoms equivalent to a $53 \mathrm{~mm}$ compressed breast for 845 mammography units of 716 services in 279 cities. The mammography units are from 13 manufacturers and 192 are screen-film technology (SFT), 544 digital computerized technology (CR) and 109 digital direct technology (DR). Average mean glandular dose $(\overline{\mathrm{MGD}})$ for SFT mammography was $1.50 \pm 0.10 \mathrm{mGy}, 2.13 \pm 0.07 \mathrm{mGy}$ for CR and $2.07 \pm 0,20$ mGy for DR mammography, with a statistically significant difference $(p<0.05)$ between conventional SFT technology and digital CR and DR technologies. It was observed no statistically significant difference when comparing $(\overline{\mathrm{MGD}})$ for $\mathrm{CR}$ and $\mathrm{DR}$ digital technologies $(\mathrm{p}=\mathbf{0 . 7 0})$. Also, $22.1 \%$ of MGDs were above the acceptable dose value of $2.50 \mathrm{mGy}$, varying between $11.6 \%$ in the South region and $31.4 \%$ in the Northeast region. Finally, the increasing number of MGD above the acceptable value with time is directly associated to the increasing number digital mammography units.

Keywords: glandular dose 1, digital mammography 2.

\section{INTRODUÇÃO}

O rastreamento do câncer de mama através da mamografia requer um rigoroso padrão técnico em sua execução e a utilização de mamógrafos com monitoramento constante de suas condições operacionais. A temática que envolve o risco de indução do câncer mamário e a qualidade do exame tem norteado uma série de diretrizes nacionais e internacionais, que visam garantir o real benefício dos exames oferecidos à população feminina [1-7].

A introdução de novas tecnologias, principalmente as digitais, permitem hoje aos fabricantes anunciarem a produção de mamógrafos que operam com baixas doses, com recursos técnicos e ferramentas computacionais de última geração para a acurácia diagnóstica [8, 9]. 
Os mamógrafos em uso no país são das tecnologias: analógica ou convencional, conhecida como sistema filme-tela intensificadora (SFT), tecnologia digital computadorizada (CR) e digital direta (DR). Na tecnologia SFT, o registro da imagem se dá através de uma combinação padronizada de filme-tela intensificadora no interior de um cassete e exige o uso de uma processadora de filmes, específica e dedicada à mamografia [1]. A tecnologia digital CR utiliza o mamógrafo convencional, uma placa de imagem (IP) usada dentro de um cassete e um digitalizador para leitura da IP sensibilizada, convertendo as informações obtidas deste processo, em imagem digital [10]. A digital direta (DR) utiliza um mamógrafo específico equipado com uma matriz de detectores que gera e registra imediatamente a imagem digital da mama [10].

Considerando o risco da indução de câncer associado à dose de radiação no tecido glandular da mama, programas que envolvem a monitoração da dose e da qualidade da imagem têm sido desenvolvidos em diversos países. Desde 1992, o Colégio Americano de Radiologia (ACR) tornou obrigatório o Programa de Acreditação em Mamografia, exigindo a certificação de todos os serviços a cada três anos, com ênfase na monitoração da dose glandular média (DGM) e da qualidade da imagem de um simulador radiográfico de mama, phantom [11, 12]. Há mais de 20 anos, a Associação Canadense de Radiologistas iniciou o Programa de Acreditação de Mamografia com o objetivo de garantir a qualidade das imagens de mamografia e monitorar a dose na mama [13].

O Colégio Brasileiro de Radiologia e Diagnóstico por Imagem (CBR), desde 1992 vêm monitorando a DGM e a qualidade das imagens de phantom e de pacientes, através do Programa de Certificação da Qualidade em Mamografia (PCQM/CBR), de caráter voluntário e que envolve custos para a certificação. O Instituto Nacional de Câncer (INCA) realizou em 2006, em parceria com o CBR, a Agência Nacional de Vigilância Sanitária (ANVISA) e o Instituto Avon, um Projeto Piloto de Qualidade em Mamografia, nos mesmos moldes do PCQM/CBR. Este projeto objetivou avaliar serviços de mamografia do Sistema Único de Saúde (SUS) em quatro Unidades da Federação. Com o êxito obtido, decorrente da metodologia desse projeto, o INCA desenvolveu em 2009, o Programa de Qualidade em Mamografia (PQM/INCA), ampliado para todos os serviços do país, também de caráter voluntário e sem custo para os participantes [14]. Desde então, o monitoramento das DGMs no país é realizado por estes dois programas.

Nos últimos anos, diversos estudos analisaram o comportamento das doses recebidas pelas mulheres, em função das tecnologias empregadas. Mora et al [15] avaliaram as DGMs para três 
espessuras de mama simuladas por placas de polimetilmetacrilato (PMMA) em mamógrafos digitais de 24 instituições, de sete países na América Latina, sendo 11 de tecnologia DR e 13 CR. Para a espessura da mama de $53 \mathrm{~mm}$, nos equipamentos DR, a média dos valores da DGM, a partir de agora representada por $\overline{\mathrm{DGM}}$, foi de $1,40 \mathrm{mGy}$, com máximo de 2,90 mGy e mínimo de 0,50 mGy. Para os equipamentos CR, a $\overline{\mathrm{DGM}}$ foi de 2,20 mGy, com máximo de 3,90 mGy e mínimo de 0,80 mGy. Os autores relataram que em 9,0\% dos equipamentos de tecnologia DR, as DGMs estavam não conformes em relação ao nível aceitável para esta espessura de mama e nos equipamentos de tecnologia CR, o mesmo ocorreu em 17,0\% dos casos. Eles concluíram que é necessário estabelecer procedimentos de otimização, principalmente para os equipamentos de tecnologia digital CR.

Timmermans et al [16] realizaram um estudo sobre o impacto na DGM resultante da mudança da tecnologia SFT para digital. Foram avaliadas as DGMs para mamas simuladas por espessuras de PMMA, em 113 unidades SFT, antes e após a digitalização para 72 unidades DR e 41 unidades CR do programa de rastreamento de câncer de mama na região de Flandres, na Bélgica, no período de 2005 a 2011. Nos sistemas SFT que foram substituídos por CR, a $\overline{\mathrm{DGM}}$ para uma mama de $53 \mathrm{~mm}$ de espessura registrou aumento de 35\%, passando de 1,40 mGy para 1,89 mGy. Nos casos em que a substituição dos sistemas SFT foi por DR, foi verificada uma redução de $26 \%$ na $\overline{\mathrm{DGM}}$, passando de 1,43 mGy para 1,06 mGy para a mesma espessura de mama.

Young e Oduko [17] estimaram no Reino Unido, no período de 2010 a 2012, as DGMs para as técnicas radiográficas que foram utilizadas nas incidências mamográficas de rotina, para mamas de várias espessuras, de 25.408 mulheres radiografadas em 359 mamógrafos de vários modelos, abrangendo as três tecnologias. Nos mesmos equipamentos foram estimadas as DGMs para a mama padrão de $53 \mathrm{~mm}$ de espessura, simuladas por placas de PMMA. Nesta espessura, para tecnologia SFT a $\overline{\mathrm{DGM}}$ foi 1,67 mGy, enquanto para CR foi 2,15 mGy e para DR 1,24 mGy. Ao comparar as $\overline{\text { DGMs }}$ das tecnologias digitais com a SFT, eles observaram aumento de 28,7\% na tecnologia CR e redução de 25,7\% na tecnologia DR. Dos 359 mamógrafos, apenas três ( $\approx 1 \%$ ) apresentaram DGM para a mama padrão acima do nível aceitável de 2,50 mGy, sendo dois de tecnologia CR e um SFT.

$\mathrm{Na}$ mesma linha de estudo, Hendrick et al [18] avaliaram a DGM em 4.366 pacientes com mama de espessura média de 53,5 mm e observaram DGMs 22,0\% menores na tecnologia DR, em comparação com a tecnologia SFT. 
Considerando os estudos mencionados acima e com intuito de prover dados sobre as doses recebidas pelas mulheres quando realizam mamografia para o rastreamento do câncer de mama no Brasil, este trabalho tem por objetivo analisar o comportamento das DGMs no período de 2011 a 2016, em função das tecnologias usadas para realizar os exames, por cada ano e região do país.

\section{MATERIAIS E MÉTODOS}

As unidades de observação foram os serviços que possuem informações sobre as suas tecnologias registradas no Sistema de Informação da Qualidade da Imagem e Interpretação Diagnóstica do INCA (QIID/INCA) e que realizaram avaliações de DGM, entre os anos de 2011 a 2016. Para a monitoração da DGM no Programa de Qualidade em Mamografia do INCA (PQM/INCA) e no Programa de Certificação da Qualidade em Mamografia do Colégio Brasileiro de Radiologia (PCQM/CBR) são enviados aos serviços de mamografia, por via postal, dois dosímetros termoluminescentes (TLD), para cada mamógrafo da instituição. Os TLDs são posicionados sobre um phantom do próprio serviço e irradiados no modo de operação automático, em cada mamógrafo [19].

A Tabela 1 mostra a distribuição dos mamógrafos existentes em cada região do país e dos mamógrafos avaliados neste trabalho.

Tabela 1: Número e percentual de mamógrafos avaliados neste trabalho e existentes, por região e total - Brasil, 2011 a 2016.

\begin{tabular}{ccccc}
\hline \multirow{2}{*}{ Região } & \multicolumn{2}{c}{$\begin{array}{c}\text { Mamógrafos avaliados } \\
\text { neste trabalho }\end{array}$} & $\begin{array}{c}\text { Mamógrafos existentes } \\
\text { no Brasil [20] }\end{array}$ \\
\cline { 2 - 5 } & $\mathbf{N}$ & $\mathbf{\%}$ & $\mathbf{N}$ & $\%$ \\
\hline Norte & 23 & 2,7 & 195 & 4,4 \\
Nordeste & 85 & 10,1 & 967 & 21,6 \\
Sudeste & 367 & 43,4 & 2.214 & 49,4 \\
Sul & 145 & 17,2 & 700 & 15,6 \\
Centro-Oeste & 225 & 26,6 & 405 & 9,0 \\
\hline Brasil & 845 & 100,0 & 4.481 & 100,0 \\
\hline
\end{tabular}


Os dados obtidos para este estudo constam na base de dados do QIID/INCA e referem-se aos registros de DGM de 608 avaliações do PQM/INCA e 507 avaliações do PCQM/CBR. Estas 1.115 avaliações de DGM foram realizadas em 845 mamógrafos, sendo 21,3\% de tecnologia SFT, 66,0\% CR e 12,7\% DR que operam em 716 serviços, de 279 cidades de todo o país. Foram coletados dados de medidas de kerma no ar de entrada na superfície do phantom $\left(\mathrm{K}_{\mathrm{i}}\right)$; tipo de tecnologia (SFT, CR e DR); e fabricantes do mamógrafo e do processador de imagem. A partir dos valores de $\mathrm{K}_{\mathrm{i}}$, foi determinada a DGM de cada avaliação e realizadas análises estatísticas do seu comportamento, em função das tecnologias, dos fabricantes dos mamógrafos e dos sistemas de processamento da imagem, para cada ano do estudo e região do país.

As informações coletadas na base de dados do QIID/INCA foram inseridas em uma planilha Excel e organizadas por: "programa que realizou a avaliação" (PCQM/CBR ou PQM/INCA); “data da avaliação"; "número do Cadastro Nacional de Estabelecimentos de Saúde (CNES) do serviço"; "localização do serviço" (Unidade da Federação e cidade); "mamógrafo" (fabricante e modelo); "tecnologia" (SFT, CR ou DR); "técnica radiográfica utilizada” (kV, mAs e combinação anodofiltro); "tipo de simulador de mama" (phantom Mama que corresponde a uma mama comprimida de $53 \mathrm{~mm}$ de espessura [21] ou phantom do Colégio Americano de Radiologia que corresponde a uma mama comprimida de $45 \mathrm{~mm}$ de espessura) [12]; “espessura da mama comprimida equivalente à espessura do simulador" (53 mm ou $45 \mathrm{~mm}$ ); e "kerma no ar de entrada na superfície ( $\mathrm{K}_{\mathrm{i}}$ )" medido com dosímetros termoluminescentes (TLD) colocados sobre os simuladores de mama. Nos casos em que a medida de $K_{i}$ foi realizada com o simulador ACR, que corresponde a uma mama de 45 $\mathrm{mm}$ de espessura, ela foi corrigida para a espessura de $53 \mathrm{~mm}$. Portanto, neste trabalho todos os valores de $\mathrm{K}_{\mathrm{i}} \mathrm{e}$, por conseguinte, da DGM são relativos à mama padrão de $53 \mathrm{~mm}$ de espessura [6].

As camadas semirredutoras (CSRs) dos feixes de raios-X utilizados para a exposição dos TLDs nas medidas de $\mathrm{K}_{\mathrm{i}}$ nos mamógrafos avaliados foram determinadas em função da técnica radiográfica empregada (kV, mAs e combinação anodo-filtro), utilizando a publicação "Dosimetry in diagnostic radiology: an international code of practice" da Agencia Internacional de Energia Atômica [22]. A DGM foi calculada para cada avaliação a partir de $\mathrm{K}_{\mathrm{i}}$ e de coeficientes de conversão utilizando a Equação 1: 


$$
D G M=K_{i} \cdot g . c . s
$$

onde: $K_{i}$ é o kerma no ar de entrada; $g$ é o coeficiente de conversão de $K_{i}$ para DGM em uma mama com $50 \%$ de glandularidade; $c$ o coeficiente que corrige a DGM para composições de mama diferentes de $50 \%$ de glandularidade; e $s$ o fator que corrige a DGM em função do uso de um espectro gerado com combinações anodo-filtro diferentes de Mo/Mo [7]. Os coeficientes $c$ e $g$ utilizados foram obtidos nas tabelas de Dance et al. [23], a partir dos dados de glandularidade da mama padrão de $53 \mathrm{~mm}$ de espessura (29\% de glandularidade) e CSR para cada técnica radiográfica. Os fatores $s$ utilizados constam da publicação "Quality Assurance Programme for Digital Mammography" da Agencia Internacional de Energia Atômica [7]. A Tabela 2 mostra os valores de DGM aceitáveis, estabelecidos nesta publicação para mamas de diversas espessuras quando comprimidas.

Tabela 2: Valores aceitáveis para a dose glandular média (DGM) [7].

\begin{tabular}{cc}
\hline $\begin{array}{c}\text { Espessura da } \\
\text { mama }(\mathbf{m m})\end{array}$ & DGM aceitável $(\mathbf{m G y})$ \\
\hline 21 & 1,00 \\
32 & 1,50 \\
45 & 2,00 \\
53 & 2,50 \\
60 & 3,00 \\
75 & 4,50 \\
90 & 6,50 \\
\hline
\end{tabular}

Os fabricantes dos mamógrafos, das processadoras de filmes radiográficos e dos digitalizadores de imagem dos sistemas CR, que apresentaram maior frequência, foram classificados nominalmente (GE, Siemens, Fuji, etc.) Os de menor frequência, foram agrupados em uma classe denominada "Outros". Foram analisadas as distribuições das DGMs por tecnologias (SFT, CR e DR), pela distribuição geográfica no país e por fabricantes e modelos dos mamógrafos e dos sistemas de processamento da imagem. 
A partir do referencial de DGM de 2,50 mGy para a mama padrão de $53 \mathrm{~mm}$ de espessura [7], foi possível identificar os casos de não conformidades deste parâmetro. Por fim, foram realizadas análises da $\overline{\mathrm{DGM}}$ para cada ano de abrangência do estudo.

O pacote estatístico SPSS (IBM SPSS Statistics 19) foi utilizado para o cálculo das variáveis derivadas e da significância estatística da diferença entre os valores das $\overline{\mathrm{DGM}}_{\mathrm{s}}$ para as tecnologias e fabricantes dos mamógrafos e dos sistemas de processamento da imagem. O SPSS também foi utilizado para avaliar o impacto temporal sobre as $\overline{\mathrm{DGM}_{\mathrm{S}}}$ nas três tecnologias. Neste trabalho, as $\overline{\mathrm{DGM}}$ s foram determinadas com intervalo de confiança de $95 \%$, por meio de duas vezes o erro padrão da média (2SEM).

\section{RESULTADOS E DISCUSSÃO}

Atualmente, existem 4.481 mamógrafos no Brasil (DATASUS) [20]. Neste estudo foram avaliados 845 mamógrafos, representando 18,9\% dos equipamentos existentes. Tendo em vista a pequena abrangência territorial dos trabalhos direcionados à avaliação das doses e tecnologias para mamografia no país [24, 25], este percentual constitui uma amostra representativa dos equipamentos instalados na maior parte das Unidades da Federação, em todas as regiões. Ao analisar esta representatividade, foram observados valores menores para o Norte e Nordeste, com os respectivos percentuais de $11,8 \%$ e $8,8 \%$ dos mamógrafos existentes nestas regiões. Por outro lado, a região Centro-Oeste apresentou o maior índice $(55,6 \%)$ de seus equipamentos avaliados e as regiões Sul e Sudeste apresentaram índices intermediários $(20,7 \%$ e $16,6 \%)$. Cabe destacar a região Centro-Oeste, que embora possua 9,0\% dos equipamentos existentes no país (405 mamógrafos), 225 foram avaliados. Este fato está relacionado com trabalhos de pesquisa realizados em parceria com as Vigilâncias Sanitárias locais, que levantaram dados na região para avaliar o impacto de um programa de qualidade em mamografia e a cobertura mamográfica [24, 25]. Nas regiões Norte e Nordeste, que possuem 1.162 mamógrafos (26\% do país), foram avaliados somente 108 equipamentos $(9,3 \%)$. Tal situação aponta para a fraca participação dos serviços nos programas de qualidade implementados no país. Nestas regiões, portanto, fica evidente a falta de ações dos 
gestores dos serviços e dos órgãos competentes, direcionadas ao controle das doses a que estão submetidas mulheres que realizam mamografia.

A Figura 1 mostra as $\overline{\mathrm{DGM}}_{\mathrm{S}}$ anuais entre 2011 e 2016 para a mama comprimida de $53 \mathrm{~mm}$ de espessura em comparação ao valor da DGM aceitável de 2,50 mGy para esta espessura de mama. Considerando todo período do estudo, a $\overline{\mathrm{DGM}}$ foi de $1,99 \pm 1,00 \mathrm{mGy}$, com uma tendência de aumento progressivo, passando de 1,71 mGy em 2011 para 2,19 mGy em 2016. Isto resultou, neste último ano, em doses 28,0\% maiores nos exames. Embora observada a tendência de aumento deste parâmetro ao longo do tempo, em nenhum ano deste estudo ele ultrapassou o valor aceitável. Em 2012, foi registrado o maior valor da $\overline{\mathrm{DGM}}$ anual, 2,29 $\mathrm{mGy}$. Dois fatores podem ter contribuído para este resultado: (a) a publicação da Portaria GM/MS n 531 de 26/03/2012 [2], que criou o Programa Nacional de Qualidade em Mamografia, ocasionando a adesão de serviços nunca antes submetidos a avaliação da dose; e (b) o alto percentual $(79,0 \%)$ de mamógrafos de tecnologia digital avaliados neste ano, como mostra a Figura 2.

Figura 1: Valor médio da dose glandular média ( $(\overline{\mathrm{DGM}})$ e nível aceitável da dose glandular média (DGM) para mama com espessura de 53 mm por ano - Brasil, 2011 a 2016.

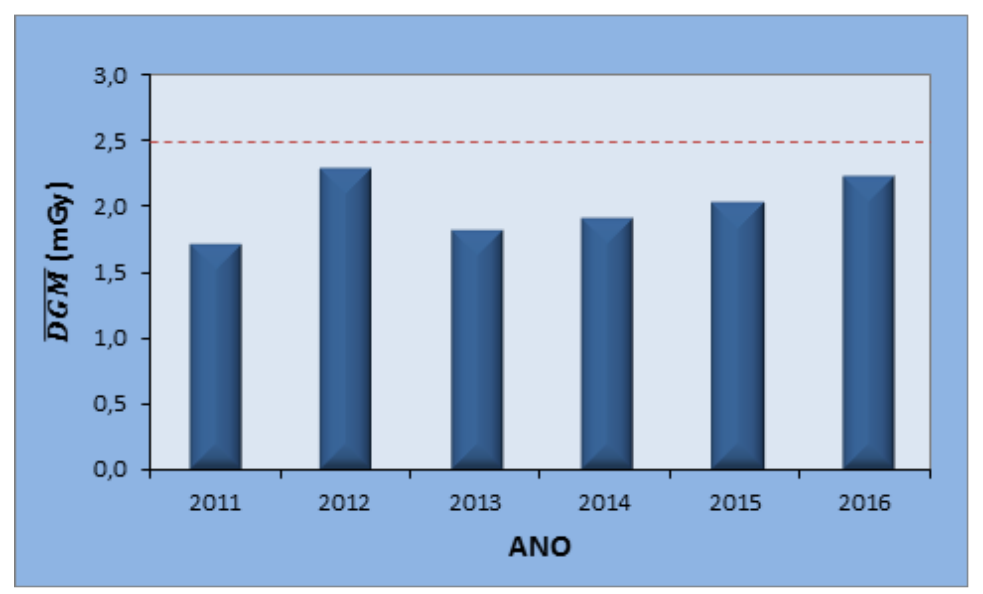

Este aumento contínuo da $\overline{\mathrm{DGM}}$ pode significar maior risco de câncer radioinduzido nas mulheres submetidas ao rastreamento do câncer de mama pela mamografia. Estudos anteriores demonstraram a importância de monitorar a DGM ao longo do tempo [17, 26] e nesse aspecto, as 
avaliações regulares realizadas nos serviços atendendo aos programas de garantia de qualidade são fundamentais.

A Tabela 3 mostra o número de avaliações e as $\overline{\text { DGM }}$ s relacionadas por tecnologia e fabricantes do mamógrafo. A $\overline{\mathrm{DGM}}$ nos mamógrafos de tecnologia SFT foi de 1,50 mGy, nos de tecnologia CR foi de 2,13 mGy e nos de tecnologia DR foi de 2,07 mGy. Houve diferença estatisticamente significativa $(\mathrm{p}<0,05)$ entre as $\overline{\mathrm{DGM}}_{\mathrm{S}}$ das tecnologias SFT e as digitais CR e DR. Entretanto, não houve diferença estatisticamente significativa $(\mathrm{p}=0,70)$ entre as $\overline{\mathrm{DGM}}$ s das tecnologias digitais $\mathrm{CR}$ e DR. Quando comparados com a $\overline{\text { DGM }}$ da tecnologia SFT, foram encontradas $\overline{\text { DGM }}$ s $42,0 \%$ e 38,0\% maiores, nas tecnologias $\mathrm{CR}$ e DR, respectivamente. Os resultados mostram que, em média, as tecnologias digitais utilizam, neste estudo, doses na mama 41,3\% maiores do que a SFT.

Tabela 3: Número e percentual de avaliações, média da dose glandular média ( $\overline{\mathrm{DGM}})$ e erro padrão da média (SEM), por tecnologia e fabricante do mamógrafo - Brasil, 2011 a 2016.

\begin{tabular}{cccc}
\hline Tecnologia & Fabricante & $\begin{array}{c}\text { Número de } \\
\text { avaliações }(\%)\end{array}$ & $\begin{array}{c}\overline{\text { DGM }} \pm \mathbf{2 S E M} \\
(\mathbf{m G y})\end{array}$ \\
\hline \multirow{5}{*}{ SFT } & GE & $79(33,3)$ & $1,48 \pm 0,12$ \\
& Hologic/Lorad & $47(19,8)$ & $1,82 \pm 0,38$ \\
& VMI & $55(23,2)$ & $1,45 \pm 0,17$ \\
& Siemens & $39(16,5)$ & $1,21 \pm 0,19$ \\
& Outros & $17(7,2)$ & $1,55 \pm 0,25$ \\
\hline \multirow{4}{*}{ CR } & Subtotal & $237(100,0)$ & $1,50 \pm 0,10$ \\
& GE & $346(47,0)$ & $2,08 \pm 0,11$ \\
& Hologic/Lorad & $216(29,3)$ & $2,37 \pm 0,12$ \\
& VMI & $51(6,9)$ & $1,92 \pm 0,29$ \\
& Siemens & $100(13,6)$ & $2,03 \pm 0,16$ \\
\cline { 2 - 4 } & Outros & $23(3,1)$ & $1,65 \pm 0,35$ \\
\hline \multirow{4}{*}{ DR } & Subtotal & $736(100,0)$ & $2,13 \pm 0,07$ \\
& GE & $31(21,8)$ & $1,59 \pm 0,24$ \\
& Hologic/Lorad & $83(58,5)$ & $2,43 \pm 0,30$ \\
& Siemens & $16(11,3)$ & $1,43 \pm 0,32$ \\
& Fuji & $12(8,5)$ & $1,63 \pm 0,47$ \\
\hline TODAS & Subtotal & $142(100,0)$ & $2,07 \pm 0,20$ \\
\hline
\end{tabular}


Ao confrontar os resultados com os já relatados na literatura para as tecnologias CR e SFT, eles seguem a mesma tendência de aumento mostrada nos trabalhos de Timmermans et al [16], Young e Oduko [17]. Entretanto, ao realizar a comparação entre as tecnologias DR e SFT, não foi encontrada a mesma tendência de redução da $\overline{\mathrm{DGM}}$ relatada por Timmermans et al [16], Young e Oduko [17] e Hendrick et al [18].

Ao analisar os resultados das $\overline{\text { DGM }}$ sem função dos fabricantes dos mamógrafos, na tecnologia SFT, os Hologic/Lorad e Siemens apresentaram diferença estatisticamente significativa $(\mathrm{p}<0,05)$, mostrando que estes últimos utilizam DGMs cerca de 34,0\% menores do que os primeiros. Quando comparada com a média dos demais fabricantes, a $\overline{\mathrm{DGM}}$ dos Hologic/Lorad foi maior em 27,6\%. Entre os fabricantes GE, VMI e "Outros" não houve diferença estatisticamente significativa (p = 0,82). Na tecnologia CR, os Hologic/Lorad apresentaram diferença estatisticamente significativa ( $p$ $<0,05)$ na $\overline{\mathrm{DGM}}$, com aumento de 16,2\% quando comparada com a média dos demais fabricantes. Nos mamógrafos de tecnologia DR foi observada a tendência de DGMs maiores nos Hologic/Lorad e Fuji, para os quais este estudo mostrou não haver diferença estatisticamente significativa $(\mathrm{p}=$ 0,05). Entretanto, Young e Oduko [17], em estudo semelhante, mostraram a tendência de DGMs maiores, nesta tecnologia, somente para os Hologic/Lorad. Quando foi realizada a comparação das $\overline{\mathrm{DGM}}_{\mathrm{S}}$ dos Hologic/Lorad e Fuji com os Siemens e GE, somente os mamógrafos Hologic/Lorad apresentaram diferença estatisticamente significativa $(\mathrm{p}<0,05)$, correspondendo a $\overline{\mathrm{DGM}}$ maior em $47,3 \%$, em relação à média dos demais fabricantes.

A Tabela 4 mostra o número de avaliações e as $\overline{\text { DGM }}$ s relacionadas por tecnologia e fabricantes da unidade de processamento: processadoras, nos casos da tecnologia SFT; e digitalizadores, nos casos da tecnologia CR. A comparação das $\overline{\mathrm{DGM}}_{\mathrm{s}}$ entre os fabricantes das processadoras de filmes radiográficos utilizadas nos sistemas de mamografia com tecnologia SFT não apresentou diferença estatisticamente significativa $(\mathrm{p}=0,61)$. O mesmo ocorreu com a comparação das $\overline{\mathrm{DGM}}$ s entre os fabricantes dos digitalizadores de imagem nos sistemas com tecnologia $C R(p=0,82)$.

Embora alguns fabricantes divulguem sistemas mais eficientes de placas de imagens no sistema CR para redução da dose $[8,27]$, isto não foi observado neste estudo. Em relação às processadoras de filmes utilizadas nos sistemas SFT, os fabricantes não costumam divulgar que haja impacto das 
suas tecnologias na redução das doses em mamografia. Os resultados deste trabalho comprovam este fato para as diversas processadoras estudadas.

Tabela 4: Número e percentual de avaliações, média da dose glandular média ( $\overline{\mathrm{DGM}})$ e erro padrão da média (SEM), por tecnologia e fabricante da unidade de processamento da imagem - Brasil, 2011 a 2016.

\begin{tabular}{cccc}
\hline Tecnologia & Fabricante & $\begin{array}{c}\text { Número de } \\
\text { avaliações } \\
(\boldsymbol{\%})\end{array}$ & $\begin{array}{c}\overline{\text { DGM }} \mathbf{2 S E M} \\
(\mathbf{m G y})\end{array}$ \\
\hline \multirow{3}{*}{ SFT } & Kodak & $90(38,0)$ & $1,54 \pm 0,21$ \\
& Macrotec & $92(38,8)$ & $1,54 \pm 0,15$ \\
& Agfa & $9(3,8)$ & $1,52 \pm 0,25$ \\
& Lotus & $15(6,3)$ & $1,39 \pm 0,27$ \\
\cline { 2 - 4 } CR & Outros & $31(13,1)$ & $1,31 \pm 0,17$ \\
\hline & Subtotal & $237(100,0)$ & $1,50 \pm 0,10$ \\
& Kodak & $327(44,4)$ & $2,13 \pm 0,11$ \\
& Agfa & $176(23,9)$ & $2,11 \pm 0,15$ \\
\cline { 2 - 4 } TODAS & Fuji & $213(28,9)$ & $2,18 \pm 0,14$ \\
& Konica & $20(2,7)$ & $1,96 \pm 0,25$ \\
\hline
\end{tabular}

O número de equipamentos avaliados, bem como as $\overline{\mathrm{DGM}}_{\mathrm{S}}$ para as mamas de $53 \mathrm{~mm}$ de espessura, nas tecnologias SFT, CR e DR e avaliação bienal entre os anos de 2011 e 2016 são mostrados na Tabela 5. A comparação das $\overline{\mathrm{DGM}}_{\mathrm{S}}$ entre os biênios em que foram realizadas as avaliações mostrou que para as três tecnologias não há diferença estatisticamente significativa, com $\mathrm{p}=0,31$ para a tecnologia SFT, $\mathrm{p}=0,54$ para a tecnologia $\mathrm{CR}$ e $\mathrm{p}=0,92$ para a tecnologia DR. Estes resultados indicam que nas três tecnologias, o período em que foi realizada a avaliação não teve impacto nas doses. Entretanto, em termos de proteção radiológica, ao longo tempo, o monitoramento da dose deve ser realizado para identificar os serviços que utilizam as DGMs mais elevadas, os quais, por conseguinte, devem otimizar as técnicas radiográficas, de modo a reduzi-las sem o comprometimento da qualidade da imagem para o diagnóstico [28]. 
Tabela 5: Número de avaliações (N), média da dose glandular média ( $\overline{\mathrm{DGM}})$ e erro padrão da média (SEM) para as mamas de $53 \mathrm{~mm}$ de espessura, por tecnologia e biênio da avaliação Brasil, 2011 a 2016.

\begin{tabular}{|c|c|c|c|}
\hline TECNOLOGIA & PERÍODO & $\mathbf{N}$ & $\overline{\mathrm{DGM}} \pm 2 \mathrm{SEM}$ \\
\hline \multirow{4}{*}{ SFT } & $2011-2012$ & 104 & $1,53 \pm 0,13$ \\
\hline & 2013-2014 & 96 & $1,38 \pm 0,20$ \\
\hline & 2015-2016 & 37 & $1,73 \pm 0,19$ \\
\hline & $2011-2016$ & 237 & $1,50 \pm 0,10$ \\
\hline \multirow{4}{*}{ CR } & 2011-2012 & 217 & $2,17 \pm 0,13$ \\
\hline & 2013-2014 & 204 & $2,08 \pm 0,12$ \\
\hline & 2015-2016 & 315 & $2,14 \pm 0,12$ \\
\hline & $2011-2016$ & 736 & $2,13 \pm 0,07$ \\
\hline \multirow{4}{*}{ DR } & 2011-2012 & 24 & $2,04 \pm 0,41$ \\
\hline & 2013-2014 & 32 & $1,99 \pm 0,15$ \\
\hline & 2015-2016 & 86 & $2,10 \pm 0,31$ \\
\hline & $2011-2016$ & 142 & $2,07 \pm 0,20$ \\
\hline TODOS & 2011-2016 & 1115 & $1,99 \pm 0,06$ \\
\hline
\end{tabular}

A tecnologia SFT vem sendo menos utilizada ao longo do tempo, com os serviços de mamografia migrando para as tecnologias digitais CR e DR. Isto indica uma mudança no parque tecnológico brasileiro, com adaptação aos modernos recursos oferecidos por estas tecnologias, como telemedicina, manipulação computacional das imagens, diagnóstico auxiliado por computador, dentre outros. Ao longo do tempo, os fabricantes destas tecnologias vêm apresentando novos modelos de equipamentos, com recursos de baixas doses (mamógrafos, detectores de imagem e softwares) $[8,9,27]$. Entretanto, na comparação entre os biênios, não foi observada influência desses recursos na redução das doses. Em contrapartida, também não foi observado aumento das doses ao longo desse período, nas três tecnologias. Isto pode ser atribuído ao trabalho de fiscalização, por meio das inspeções periódicas nos serviços [1] e aos programas de qualidade em mamografia implementados no país [3,19], já que ambos contribuem significativamente neste aspecto.

A Figura 2 mostra para cada ano do estudo, o percentual de DGM não conformes (acima de 2,50 mGy), considerando todas as tecnologias e o percentual de equipamentos digitais (CR e DR) avaliados. Entre os anos de 2011 e 2016 houve um aumento de 50\% dos equipamentos digitais 
avaliados. Também foi observado o aumento no percentual de não conformidades da DGM de 10,6\% em 2011 para 29,4\% em 2016, o que representou um aumento de $176 \%$ em todo o período.

Figura 2: Percentual de dose glandular média (DGM) não conforme em todas as tecnologias e percentual de equipamentos digitais (CR e DR), por ano - Brasil, 2011 a 2016.

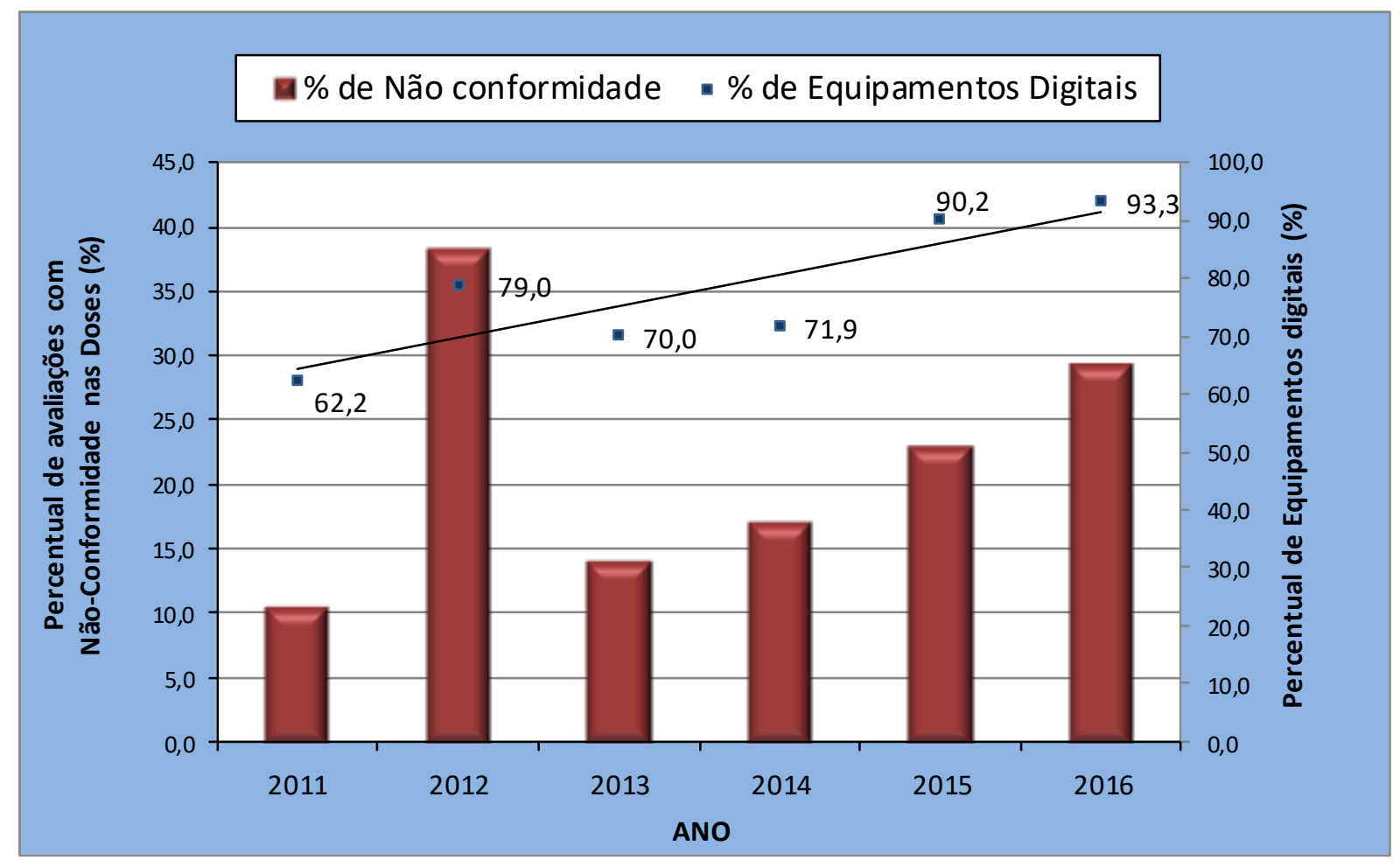

Pode ser destacado o ano de 2012, quando o percentual de não conformidades atingiu o máximo de $38,2 \%$. Os motivos para a ocorrência deste resultado são os mesmos que levaram ao maior valor da $\overline{\mathrm{DGM}}$ neste mesmo ano, apresentados na Figura 1 e discutidos anteriormente. $\mathrm{O}$ aumento das não conformidades apresenta relação direta com o aumento dos equipamentos de tecnologia digital. Young e Oduko [17] no Reino Unido, observaram que a tecnologia CR apresentou DGMs mais elevadas, embora somente $1 \%$ de todos os equipamentos tenham apresentado valores fora do nível aceitável. É importante ressaltar que a boa prática em mamografia recomenda que este nível nunca seja ultrapassado $[4,7]$. 
Na Tabela 6 são apresentados o número e o percentual de avaliações e das não conformidades da DGM, por região do país. Em um total de 1.115 avaliações, 246 foram consideradas não conformes, ou seja, 22,1\% estavam acima do valor aceitável de 2,50 mGy.

Tabela 6. Número e percentual de avaliações da dose glandular média (DGM) realizadas e número e percentual de avaliações da DGM consideradas não conformes, por região e total no Brasil - 2011 a 2016.

\begin{tabular}{ccccc}
\hline Região & \multicolumn{2}{c}{$\begin{array}{c}\text { Avaliações da DGM } \\
\text { realizadas neste trabalho }\end{array}$} & \multicolumn{2}{c}{$\begin{array}{c}\text { Avaliações da DGM } \\
\text { consideradas não } \\
\text { conformes }\end{array}$} \\
\cline { 2 - 5 } & $\mathbf{N}$ & $\mathbf{\%}$ & $\mathbf{N}$ & $\mathbf{\%}$ \\
\hline Norte & 31 & 2,8 & 8 & 25,8 \\
Nordeste & 118 & 10,6 & 37 & 31,4 \\
Sudeste & 492 & 44,1 & 120 & 24,4 \\
Sul & 172 & 15,4 & 20 & 11,6 \\
Centro-Oeste & 302 & 27,1 & 60 & 19,9 \\
\hline Brasil & 1.115 & 100,0 & 246 & 22,1 \\
\hline
\end{tabular}

Embora as regiões Nordeste e Sul tenham apresentado, respectivamente, o maior e o menor índice de não conformidades nas avaliações, realizadas por cada região, isto representou 15,0\% e $8,1 \%$ de todas as avaliações não conformes do estudo. $\mathrm{O}$ maior número de avaliações realizadas ocorreu na região Sudeste, com o percentual de não conformidades próximo ao percentual médio de todas as não conformidades encontradas. As avaliações não conformes desta região corresponderam a 48,8\% de todas as avaliações não conformes do estudo. Por outro lado, a região Norte, mesmo com menor representatividade de avaliações, apresentou percentual de não conformidades em seus equipamentos, maior do que a região Sudeste, embora representando apenas 3,3\% do total de avaliações não conformes do estudo.

O número de avaliações realizadas neste estudo mostrou um quadro significativo do comportamento das DGMs em todas as regiões do país. No estudo de Mora et al [15], com dados coletados entre 2012 e 2014 de diversos países da América Latina, a amostra utilizada para avaliar a DGM no Brasil representou apenas 0,11\% dos equipamentos do país. Correa [29], em 2010, avaliou a DGM em 100 equipamentos no estado de Goiás, o que representou, na época, 94,3\% dos 
equipamentos do Estado e 2,3\% dos existentes no país. Apesar da importância destes estudos, suas amostras foram pouco representativas em relação ao território nacional.

\section{CONCLUSÕES}

A distribuição por região dos mamógrafos avaliados na amostra do estudo é representativa da distribuição por região dos mamógrafos do país. A média anual da DGM em nenhum ano ultrapassou o valor aceitável de 2,50 mGy para mama de $53 \mathrm{~mm}$ de espessura; no entanto, foi observado aumento da $\overline{\mathrm{DGM}}$ ao longo do tempo.

As $\overline{\mathrm{DGM}}_{\mathrm{S}}$ para as tecnologias digitais CR e DR foram $42,0 \%$ e $38,0 \%$ maiores do que para a tecnologia SFT. Considerando os fabricantes, concluiu-se que os mamógrafos da Hologic/Lorad trabalham com $\overline{\mathrm{DGM}}_{\mathrm{s}}$ mais altas nas três tecnologias (SFT, CR e DR) e que as diversas marcas de

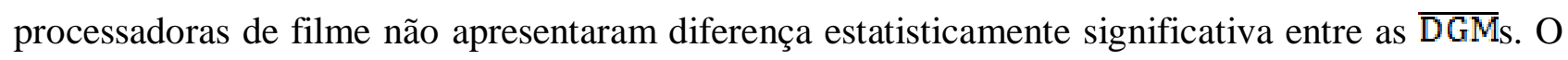
mesmo ocorreu entre os fabricantes de digitalizadores CR. Também foi observado que, ao longo do tempo, os avanços tecnológicos e recursos de baixas doses introduzidos tanto na tecnologia CR, como na DR, não tiveram impacto na $\overline{\mathrm{DGM}}$.

Em 22,1\% das estimativas de dose, o valor de DGM estava acima do valor aceitável de 2,50 mGy. Isto indica a necessidade de maior participação dos serviços nos programas nacionais de qualidade e de intensificação das inspeções periódicas pelos órgãos fiscalizadores, que verificam se as doses estão dentro deste valor aceitável, cobrando a sua adequação, quando necessário. Adicionalmente, intervenções e tomadas de decisões no âmbito dos próprios serviços, com base nos princípios de Proteção Radiológica e em métodos de Gestão da Qualidade podem garantir doses dentro dos níveis aceitáveis em todos os exames realizados.

$\mathrm{O}$ aumento anual das não conformidades apresentou relação direta com o aumento do número de mamógrafos de tecnologia digital CR e DR em substituição aos de tecnologia SFT, o que implica no aumento do risco de câncer radioinduzido para as mulheres que realizam exames nestes equipamentos. 


\section{AGRADECIMENTO}

Agradecemos a todas as pessoas que se dedicaram de alguma forma para colaborar com este estudo, aos órgãos de fomento do Programa de Capacitação Institucional que proporcionaram o projeto número 300156/2017-0 e garantiram esta publicação.

\section{REFERÊNCIAS}

[1] BRASIL, Ministério da Saúde, Agência Nacional de Vigilância Sanitária. Portaria no 453, de 01 de junho de 1998, aprova o Regulamento Técnico que estabelece as Diretrizes Básicas de Proteção Radiológica em Radiodiagnóstico Médico e Odontológico. Diário oficial [da Republica Federativa do Brasil]. Brasilia, 1998.

[2] BRASIL, Ministério da Saúde, Gabinete do Ministro. Portaria GM/MS no 531, de 26 de março de 2012. Institui o Programa Nacional de Qualidade em Mamografia (PNQM), Diário Oficial da União, no 60, Seção 1, Brasília, 2012.

[3] BRASIL, Ministério da Saúde, Gabinete do Ministro. Portaria GM/MS no 2898, de 28 de novembro de 2013. Revoga a Portaria GM/MS n 531, de 26 de março de 2012 e atualiza o Programa Nacional de Qualidade em Mamografia (PNQM), Diário Oficial da União, no 60, Seção 1, Brasília, 2013.

[4] PERRY N., BROEDERS M., DE WOLF C., ET AL. European guidelines for quality assurance in breast cancer screening and diagnosis. 4th ed. Luxembourg: European Communities, 2006. Disponível em: http://ec.europa.eu/health/ph_projects/2002/ cancer/fp_cancer_2002_ext_guid_01.pdf. Consultado em 01/11/2017.

[5] IAEA - International Atomic Energy Agency, Control de Calidad en Mamografia, Protocolo elaborado en el marco de dos proyetos regionals ARCAL/IAEA, TECDOC-1517, Vienna, 2006. Disponível em: https://www-pub.iaea.org/MTCD/Publications/PDF/te_1517s_web. pdf. Consultado em 01/11/2017. 
[6] IAEA - International Atomic Energy Agency, Quality Assurance Programme for Screen Film Mammography, Human Health Series no 2, Vienna, 2009. Disponível em: https://wwwpub.iaea.org/MTCD/Publications/PDF/Pub1381 web.pdf. Consultado em 01/11/2017.

[7] IAEA - International Atomic Energy Agency, Quality assurance programme for digital mammography. Human health series No. 17. Vienna, 2011. Disponível em: http://wwwPub.iaea.org/MTCD/publications/PDF/Pub1482 web.pdf. Consultado em 30/09/2014.

[8] FUJIFILM. Disponível em: http://www.fujifilmamericas.com.br/press/news/display_news ? newsID=880779. Consultado em 9/11/2017.

[9] MICRODOSE SI Sistema de Mamografia. Disponível em: https://www.philips.com.br/ healthcare/product/HC714045/microdose-mammography Consultado em 02/02/2018.

[10] SANTOS, A. F., 2010, Física Médica em Mamografia, $1^{\text {a }}$ edição, Rio de Janeiro, Editora Revinter.

[11] AMERICAN COLlEGE OF RADIOLOGY, Mammography Accreditation Program Requirements. Disponível em: http://www.acraccreditation.org/ /media/ ACRAccreditation/Documents/Mammography/Requirements.pdf?la=en. Consultado em $01 / 11 / 2017$.

[12] DeStOUET J. M., BASSETT L. W., YAFFE M. J., BUTLER P. F., WILCOX P. A., The ACR's Mammography Accreditation Program: Ten Years of Experience Since MQSA. J Am Coll Radiol 2005; 2:585-594.

[13] CANADIAN ASSOCIATION RADIOLOGISTS, Mammography Accreditation Program.

Disponível em: http://www.car.ca/en/accreditation/map.aspx. Consultado em 01/11/2017.

[14] INCA - Instituto Nacional do Cancer, Programa - Controle de Cancer de Mama: Qualidade em Mamografia. Disponível em: http://www2.inca.gov.br/wps/wcm/connect/acoes programas/site/home/nobrasil/programa_controle_cancer_mama/qualidade_mamografia.

Consultado em 15/08/2017.

[15] MORA P., KHOURY H., BITELlI R., QUINTERO A.R., GARAY F., AGUILAR J.G., GAMARRA M. AND UBEDA C., Latin American Image Quality Survey in Digital Mammography Studies, Radiation Protection Dosimetry 2017, Vol. 174, No. 1, pp. 94-101. 
[16] TIMMERMANS L., DE HAUWERE A., BACHER K., BOSMANS H., LEMMENS K., BLEYEN L., VAN LIMBERGEN E., MARTENS P., VAN STEEN A., MORTIER G., VAN HERCK K., THIERENS H., Impact of the digitalisation of mammography on performance parameters and breast dose in the Flemish Breast Cancer Screening Programme. Eur Radiol. 2014; 24(8):1808-19. doi: 10.1007/s00330-014-3169-y. Disponível em: https://www.ncbi.nlm.nih.gov/pubmed/ 24816 932. Consultado em 01/11/2017.

[17] YOUNG K.C., ODUKO J.M., Radiation doses received in the United Kingdom breast screening programme in 2010 to 2012. Br J Radiol 2016; 89: doi: 10.1259/bjr.20150831.

[18] HENDRICK R.E., PISANO E.D., AVERBUKH A., ET AL., 2010, Comparison of Acquisition Parameters and Breast Dose in Digital Mammography and Screen-Film Mammography in the American College of Radiology Imaging Network Digital Mammographic Imaging Screening Trial. AJR Am J Roentgenol. 2010; 194(2): 362-369.

[19] COLÉGIO BRASILEIRO DE RADIOLOGIA E DIAGNÓSTICO POR IMAGEM, Programa de qualidade em mamografia, Normas Básicas para Inscrição no Programa de Qualidade em Mamografia. Disponível em: http://www.praxis.med.br/pasta downloads/FolderMama.pdf. Consultado em 01/11/2017.

[20] CNES/DATASUS - Cadastro Nacional de Estabelecimentos de Saúde/Departamento de Informática do SUS, 2017, Consulta Equipamentos, disponível em: http://datasus. saude.gov.br/cadastro-nacional-de-estabelecimentos-de-saude. Consultado em 04/10/2017.

[21] PEIXOTO, J.E. ; ALMEIDA, C.D. ; KOCH H.A. Manual de Instruções para as Medidas de Controle de Qualidade dos parâmetros técnicos da mamografia. Rio de Janeiro: SCMRJ, 1998 (Nota técnica).

[22] IAEA - International Atomic Energy Agency, Dosimetry in diagnostic radiology: an international code of practice. IAEA technical report series 457, IAEA, Vienna, 2007. Disponível em: http://www-pub.iaea.org/MTCD/publications/PDF/TRS457 web.pdf. Consultado em 30/09/2017.

[23] DANCE D.R., SKINNER C.L., YOUNG K.C., BECKETT J.R., KOTRE C.J. Additional factors for the estimation of mean glandular breast dose using the UK mammography dosimetry protocol. Phys Med Biol. 2000; 45:3225-40. 
[24] CORRÊA R.S., PEIXOTO J.E., SILVER L.D., DIAS C.M., NOGUEIRA M.S., HWANG S.F., ET AL. Impacto de um Programa de avaliação da qualidade da imagem nos serviços de mamografia do Distrito Federal. Radiol Bras 2008; 41:109-14.

[25] CORRÊA R.S., JÚNIOR R.F., PEIXOTO J.E., RODRIGUES D.C.N., LEMOS M.E.F., MARINS L.A.P., SILVEIRA E.A., Estimativas da cobertura mamográfica no Estado de Goiás, Brasil, Cad. Saúde Pública, Rio de Janeiro, 27(9):1757-1767, 2011.

[26] DE HAUWERE A., THIERENS H., Impact of digitalization of mammographic units on average glandular doses in the Flemish Breast Cancer Screening Programme. Breast ImagingProceedings $11^{\text {th }}$ International Workshop, IWDM 2012, Philadelphia, pp 410-417.

[27] INOVAÇÕES CR AGFA. Disponível em: http://www2.ibf.com.br/medix/crs/dx-m/?lang=pb Consultado em 02/02/2018.

[28] ALMEIDA, C.D., Otimização da dose glandular média na mama e da qualidade da imagem nos sistemas de mamografia digital, Tese (Doutorado em medicina, Radiodiagnóstico) HUCFF/UFRJ, Rio de Janeiro, Brasil (2014).

[29] CORREA, R.S., Mamografia: Infraestrutura, Cobertura, Qualidade e Risco do Câncer Radioinduzido em Rastreamento Oportunístico no Estado de Goiás, Tese (Doutorado em Ciências da Saúde) UFG, Goiás, Brasil (2012). 